\title{
AVALIAR O CONHECIMENTO SOBRE INTOXICAÇÃO INFANTIL NO MUNICÍPIO DE PRESIDENTE PRUDENTE (SP)
}

Aline Cargnin Marcelino; Guilherme Gomes dos Santos Alves; Gabriela Tavernaro Tsakamoto; João Victor Fuzeta Peres; Mariane Redivo Aljonas; Mariele Redivo Aljonas; Otávio Frederico de Toledo; Wadih Rodrigues Assunção Vilela Amado; Angélica Augusta Grigoli-Dominato

Universidade do Oeste Paulista - UNOESTE, Curso de Medicina, Presidente Prudente, SP. E-mail: angelica@unoeste.br

\section{RESUMO}

Intoxicação infantil ocorre diariamente, com as diferentes classes químicas de agentes tóxicos. O objetivo do estudo foi identificar o conhecimento sobre intoxicação infantil. A metodologia utilizada foi a aplicação de questionários antes e após a entrega de folder explicativo, após a anuência do TCLE. Nos resultados observou-se que 30\% dos entrevistados tinham breve conhecimento sobre o assunto. O nível de conhecimento e o contato com o assunto passou de 60,3 \% para 86,9 \% após a apresentação do folder explicativo. Declararam a ocorrência de 10,2\% de casos de intoxicação infantil, principalmente nas próprias residências. Relataram que os medicamentos apresentam $94 \%$ de riscos em causar intoxicações. Os riscos existem, entretanto, podem ser controlados, quando os agentes tóxicos são armazenados de forma correta e consciente. Divulgar e orientar o sobre as intoxicações é uma forma de minimizar o número de episódios assim como sua gravidade.

Palavras-Chaves: intoxicações infantis, exposição química, eventos toxicológicos, intoxicação aguda, medicamentos,

\section{ASSESSING KNOWLEDGE OF POISONING IN CHILDREN PRESIDENTE PRUDENTE (SP)}

\begin{abstract}
Child poisoning occurs daily, with different classes of chemicals toxic agents. The objective of the study was to identify the knowledge about child poisoning. The utilized methodology was the application of questionnaires before and after the delivery of an explanatory folder, after the consent of the written informed consent form (WICF). In the results was possible to observe that $30 \%$ of the interviewee had brief knowledge about the subject matter. The level of knowledge and contact with the subject matter increased from $60,3 \%$ to $86,9 \%$ after a presentation of the explanatory folder. Declare the occurrence of $10,2 \%$ of poisoning child cases, mainly in their own house. Reported that the medicines presented $94 \%$ of risk to cause poisoning. The risks exist, however, can be controlled when the toxic agents are correctly and consciously stored. To publish and to advise about the poisoning is a way to minimize the numbers of poisoning cases as well its severity.
\end{abstract}

Key Words: child poisoning, chemical exposure, toxicological events, acute intoxication, medicines. 


\section{INTRODUÇÃO}

Intoxicação é a interação do agente tóxico, de qualquer natureza, com o organismo gerando um conjunto de efeitos orgânicos, denominados sinais e sintomas ${ }^{1,2}$. No Brasil, anualmente são notificadas as ocorrências de milhares de casos, com as diversas classes de produtos químicos, como medicamentos, alimentos contaminados, agrotóxicos, animais peçonhentos e entre outras substâncias químicas ${ }^{3}$.

A intoxicação infantil tem maior incidência em crianças menores de 5 anos de idade, por serem movidas pela curiosidade, além de estarem na fase de aprimoramento do desenvolvimento motor. Com isso são capazes de alcançar e manusear objetos com maior habilidade, como abertura de embalagens. O ambiente familiar trata-se de outro fator associado, pois os pais ou responsáveis subestimam os riscos de exposição e intoxicação ${ }^{4}$.

Muitos fatores colaboram com as exposições, por exemplo, embalagens e conteúdos coloridos dos produtos químicos, com formatos diferentes, odores frutais, medicamentos com sabores agradáveis. O armazenamento em locais de fácil acesso e/ou acondicionados irregularmente em embalagens de alimentos ou bebidas facilita o acesso e aumentam os riscos de intoxicações ${ }^{5,6}$.

Os medicamentos são os agentes tóxicos mais comuns em causar intoxicações infantis devido às embalagens atrativas, ausência de embalagem especial, com trava, para proteger do manuseio da criança e a prática da automedicação são fatores que contribuem para o risco da intoxicação em crianças ${ }^{4}$.

O desenvolvimento cognitivo e o avançar da idade diminuem os casos acidentais de intoxicação domiciliar. Entretanto, crianças maiores de 5 anos estão também expostas aos animais peçonhentos, plantas tóxicas, alimentos e erros terapêuticos ${ }^{7}$.

No Brasil em 2012, os casos registrados de intoxicação prevaleceram na faixa etária entre 1 e 4 anos, sendo que os medicamentos foram os agentes mais comuns (36,9\%), seguidos dos domissanitários $(20,3 \%)$ e produtos químicos industriais $(9,3 \%)^{8,9}$.

Entre os anos de 2010 a 2015, foram registrados no DATASUS, 74.589 casos de intoxicações exógenas em crianças de 0 a 9 anos de idade, sendo que na faixa etária entre 1 a 4 anos. Entre os gêneros, há maior prevalência do sexo masculino. Os produtos mais citados nos registros de intoxicações infantis, neste período foram os medicamentos com 27.514 casos, seguidos dos produtos de uso domiciliar com 12.043, e alimentos e bebidas com $6.934^{10}$.

O objetivo do estudo foi levantar a ocorrência de casos de intoxicações agudas em crianças, no município de Presidente Prudente (SP) e os principais agentes tóxicos causadores.

\section{METODOLOGIA}

O projeto foi submetido e aprovado no Sistema Gestor de Pesquisa sob o protocolo número 3082 e Comitê de Ética CAAE 53952616.9.0000.5515.

O método utilizado foi a aplicação de questionários para as mães ou responsáveis legais que convivem com crianças. A abordagem dos voluntários foi realizada em uma "sala de espera" de um laboratório de Análises Clínicas, de uma universidade, que faz o atendimento de diferentes convênios, no município de Presidente Prudente (SP). Os participantes convidados, inicialmente assinaram o TCLE e posteriormente responderam ao questionário que continha 23 questões. Após esta primeira abordagem foi entregue, a cada voluntário, um folder ilustrativo e explicativo sobre os riscos de intoxicações infantis, o armazenamento correto de medicamentos e produtos higiênico-sanitários, praguicidas, plantas tóxicas e acidentes com animais peçonhentos. Em seguida, os voluntários responderam novamente ao mesmo questionário, para que as respostas, do antes e depois do recebimento das informações contidas no folder, fossem comparadas. 


\section{RESULTADOS}

O estudo totalizou 100 voluntários que responderam duas vezes ao mesmo questionário, antes e após a apresentação de folder explicativo sobre riscos de intoxicação infantil. Permitiu observar diversas reações dos voluntários frente à fragilidade, vulnerabilidade e riscos reais de intoxicações infantis. Um fator marcante foi que $70 \%$ dos entrevistados (Figura 1) desconheciam por completo sobre intoxicações exógenas, sendo que, o folder apresentado foi o primeiro contato com o assunto.

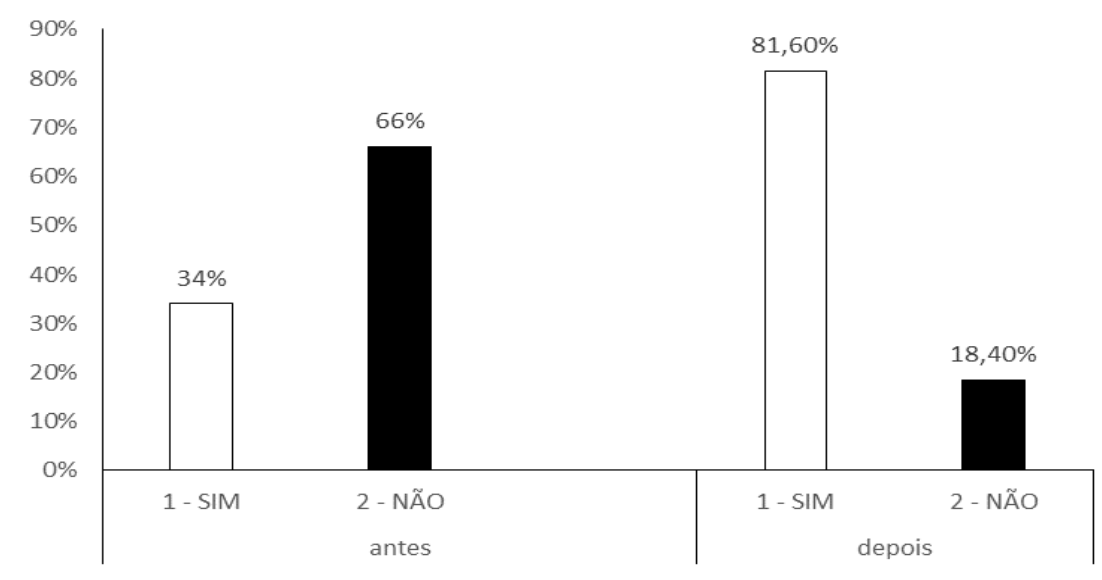

Figura 01. Nível de conhecimento sobre as intoxicações exógenas com crianças, antes e depois da apresentação do folder.

Dentre aqueles que responderam conhecer os riscos da intoxicação infantil, $20 \%$ relataram que a informação foi prestada pelo pediatra, que é profissional capacitado para fornecer as informações corretamente.

A Figura 2 mostra o nível de conhecimento sobre o significado de substância tóxica. Observou-se que antes do folder 39,4\% relataram desconhecimento, entretanto após a entrega do $13,1 \%$ dos entrevistados disseram ainda desconhecer tal assunto, no entanto relataram a ocorrência de 10,2\% de intoxicações infantis.

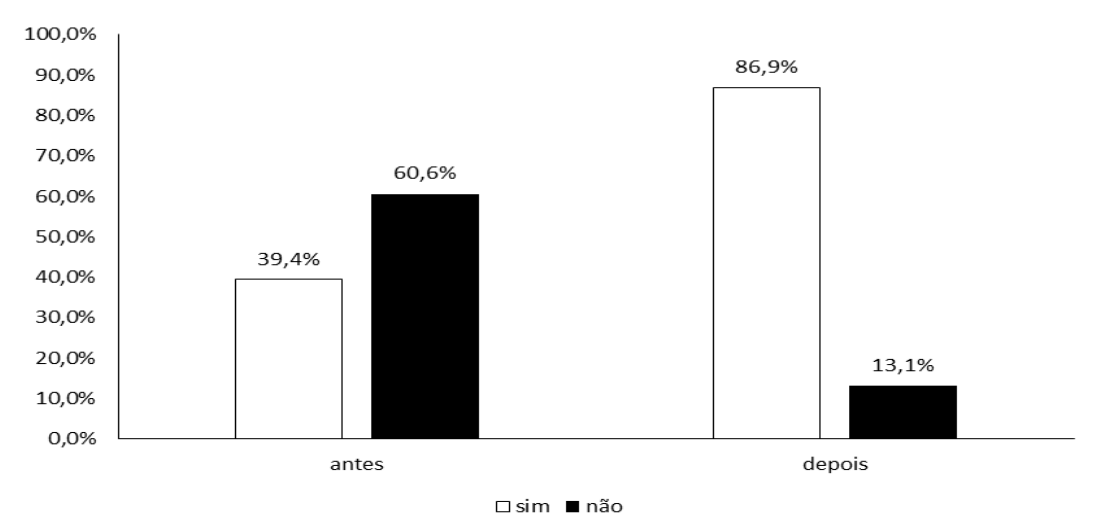

Figura 02. Nível de conhecimento dos voluntários sobre intoxicações infatis, antes e após o folder Ao serem questionados quanto à busca de auxílio nos casos de intoxicações, $96 \%$ relataram que procurariam atendimento especializado ou seja, pediatra e emergência, entretanto, inicialmente, $3 \%$ disseram que recorreriam ao vizinho. 
Tabela 01. Obtenção de informações sobre intoxicações infantis antes e após o folder

\begin{tabular}{lll}
\hline Obtenção da informação & \multicolumn{2}{l}{ Respostas (\%) } \\
& Antes & Após \\
\hline TV & 18 & 19 \\
Rádio & 5 & 4 \\
Vizinho & 5 & 4 \\
Revista/Jornal & 7 & 6 \\
Médico & 5 & 21 \\
Outro & 0 & 32 \\
\hline
\end{tabular}

A Tabela 1 mostra que antes de receber as informações do folder, os programas de televisão eram os responsáveis por tais informes, entretanto após o contato com o folder, 53\% relatou ciência sobre o referido assunto.

Tabela 02. Nível de conhecimento, antes e depois da entrega do folder, sobre os locais ideais de armazenamento dos produtos de limpeza e medicamentos no interior das residências

\begin{tabular}{l|cc|cc}
\hline & \multicolumn{2}{|c|}{ Produtos de limpeza } & \multicolumn{2}{c}{ Medicamentos } \\
Locais & Antes (\%) & Depois (\%) & Antes (\%) & Depois (\%) \\
\hline Balcão da cozinha & 28 & 30 & 22 & 23 \\
Armário aéreo & 06 & 16 & 03 & 04 \\
Balcão do banheiro & 10 & 08 & 09 & 11 \\
Prateleira & 24 & 22 & 27 & 27 \\
Armário aéreo do banheiro & 04 & 06 & 17 & 16 \\
Outros & 28 & 18 & 22 & 19 \\
\hline Acesso das crianças & Antes (\%) & Depois (\%) & Antes (\%) & Depois (\%) \\
Sim & 9 & 14 & 07 & 08 \\
Não & 91 & 86 & 93 & 92 \\
\hline
\end{tabular}

Entretanto observa-se na Tabela 2 que os produtos de limpeza e medicamentos são armazenados, principalmente, em balcões do banheiro, da cozinha e prateleiras. Esses dados foram identificados nas respostas de $17 \%$ dos participantes antes da apresentação do folder contra $4 \%$ após a entrega do mesmo.

No estudo os participantes (Tabela 3), relataram que 17,2\% não conheciam os riscos de intoxicações por medicamentos, antes de receber as informações através do folder explicativo. Entretanto, 82,8\% disseram estar cientes dos problemas causados pelos medicamentos, este número passou para $95 \%$ após a apresentação do folder.

Dentre os participantes 30\% relataram a ocorrência de episódios de intoxicação, na família, sendo que, $5 \%$ aconteceu com os filhos, $7 \%$ nas suas próprias residências e $2 \%$ na casa dos avós. Quando questionados a quem recorreriam em casos de intoxicação, $96 \%$ responderam que buscariam atendimento médico, sendo $22 \%$ a procura ao pediatra e $74 \%$ de atendimento hospitalar. 
Tabela 03. Conhecimento sobre as intoxicações por medicamentos e a quem recorrer nos eventos toxicológicos

\begin{tabular}{lcc}
\hline \multicolumn{3}{c}{ Riscos de intoxicação por medicamentos } \\
\hline Sim & Antes (\%) & Depois (\%) \\
Não & 82,8 & 95,0 \\
\hline
\end{tabular}

\section{DISCUSSÃO}

Desconhecer o assunto intoxicação infantil e os riscos de suas causas refletem no grande número de casos de acidentes domésticos de intoxicação ocorridos no Brasil.

As intoxicações exógenas acontecem por uma série de situações, que quando sincronizadas aumentam a possibilidade de ocorrência. Dentre as circunstâncias mais comuns estão armazenamento e acondicionamento incorreto dos agentes tóxicos, pouca vigilância, tentativas de suicídio e homicídio, entre outras. As crianças são mais susceptíveis por causa da curiosidade, própria da idade, na descoberta do mundo que as cercam. Entre os agentes comumente causadores de intoxicação estão os medicamentos com $45 \%$ dos casos de intoxicações entre os adultos, entretanto entre crianças menores de 10 anos de idade medicamentos e domissaneantes estavam em $37,2 \%$ e $37,9 \%$, respectivamente ${ }^{11}$. Por isso, faz-se necessária a divulgação sobre os riscos de intoxicações com produtos químicos e medicamentos contidos no interior das residências.

Este fato, reforça a importância da atenção primária à saúde, e sua contribuição para maior consciência da população, diminuindo assim, o índice de intoxicação entre as crianças. Após a apresentação do folder, a maioria dos entrevistados entenderam sobre a importância de obtenção de informações corretas, assim como condutas dos tratamentos prestados pelos profissionais capacitados. Para diminuir ou eliminar os riscos de intoxicações com crianças esses resultados deveriam estar próximos ou iguais a $100 \%$ de conhecimento sobre o assunto.

Crianças com 4 anos de idade são mais susceptíveis às intoxicações devido à peculiaridade do desenvolvimento infantil, contabilizando $81 \%$ dos casos. Da totalidade de casos, os agentes tóxicos mais comuns são medicamentos (35\%) e produtos químicos (17\%).

Em estudo realizado $29 \%$ dos casos de intoxicações infantis ocorreram com crianças, sendo o agente tóxico responsável o medicamento ${ }^{12,13}$. Então, a predisposição da criança aos agentes tóxicos, está intimamente relacionada ao acesso à informação e ao conhecimento dos adultos responsáveis pela sua segurança.

Cerca de $18,2 \%$ dos casos de intoxicações medicamentosas, ocorreram entre crianças de zero a 9 anos de idade, sendo que $11,6 \%$ estavam na faixa etária entre 1 a 4 anos ${ }^{14}$. Sendo assim, o armazenamento dos agentes tóxicos nas residências é um dos fatores mais importantes para o desencadeamento das intoxicações infantis. Os locais ideais, que inibem o acesso da criança são armários aéreos com portas e travas que dificultem sua abertura.

Entre os anos de 2014 e 2015 foram notificados 2 casos de intoxicações com crianças na faixa etária de zero a 9 anos de idade ${ }^{10,15}$. Esses valores não correspondem àqueles encontrados no estudo, o que sugere a ocorrência de subnotificações.

\section{CONCLUSÃO}

O conhecimento da intoxicação infantil é pequeno frente à população em geral, em especial as mães do Município de Presidente Prudente. A própria residência permanece como local de ocorrência dos envenenamentos. Levando-se em consideração esses aspectos faz-se necessário uma melhor abordagem sobre os perigos e procedimentos diante de qualquer exposição aos agentes tóxicos. Por esse motivo é de grande importância que a população esteja consciente e em alerta sobre o assunto. 


\section{REFERÊNCIAS}

Larini L. Toxicologia 2 ed. São Paulo. Editora Manole. 1993.

Zambolim CM, Oliveira TP, Hoffmann NA, Vilela CE, Neves D, Dos Anjos FR, Soares LM, Tiburzio LS, Cardoso AF, Murad MB, Magalhães MG, Oppermann PER, Guimarães STJ. Perfil das intoxicações exógenas em um hospital universitário. Revista Médica de Minas Gerais 2008; 18(1): 5-10

Brasil - Agência Nacional de Vigilância Sanitária e Instituto de Defesa do Consumidor [homepage on the Internet]. Vigilância sanitária, alimentos, medicamentos, produtos e serviços de interesse a saúde - guia didático, 2007 [cited 2014 Apr]. [acesso em 20 junho 2016]. Disponível em http://pesquisa.bvsalud.org/sms/resource/pt/sms-1274

Lourenço J, Furtado BMA, Bonfim C, Intoxicações exógenas em crianças atendidas em uma unidade de emergência pediátrica, Revista Acta Paulista Enfermagem 2008; 21(2):282-286. https://doi.org/10.1590/S0103-21002008000200008

Grigoli-Dominato, A. A. Levantamento estatístico das intoxicações exógenas relatadas por indivíduos atendidos em uma UBS do município de Presidente Prudente (SP) Colloquium Vitae 2012; 4: 31-38

Presgrav RF, Camacho LA, Villas Boas MH Analysis of data from Poison Control Centers in Rio de Janeiro, Brazil, for use in public health activities. Cad Saúde Pública 2009; 25 (25): 401-408

Oliveira FFS, Aparecida, E; Epidemiological profile of exogenous poisoning in children and adolescents from a municipality in the state of Mato Grosso, Revista Paulista de Pediatria 2014; 32 (4): 299-305. https://doi.org/10.1016/i.rpped.2014.06.002

FioCruz. Disponível em:

<http://www.fiocruz.br/sinitox/media/Tabela\%207 2012.pdf>. [Acesso em: 21 junho. 2015]

Fook SML, Azevedo EF, Costa MM, Feitosa ILF, Bragagnoli G, Mariz SR Avaliação das intoxicações por domissanitários em uma cidade do Nordeste do Brasil, Cadernos Saúde Pública, Rio de Janeiro 2013; 29 (5): 1041-1045

DataSus. (2015) Disponível em:

<http://tabnet.datasus.gov.br/cgi/deftohtm.exe?sinannet/cnv/Intoxbr.def> . Acesso em: $10 \mathrm{de}$ Abril, 2016

Werneck GL, Hasselmann MH Intoxicações exógenas em hospitais da região metropolitana do Rio de Janeiro. Cadernos Saúde Coletiva 2005; 13 (3): 767-778

Tavares EO, Buriola AA, Santos JAT, Ballani TSL, Oliveira MLF Fatores associados à intoxicação infantil. Escola Anna Nery 2013; 17 (1): 31-37 https://doi.org/10.1590/S1414-81452013000100005.

Siqueira KM, Brandão JM, Lima HF, Garcia ACA, Gratone FM, Brasileiro MSE Perfil das intoxicações exógenas infantis atendidas em um hospital especializado da rede pública de Goiânia-GO. Revista Eletrônica de Enfermagem 2008; 10 (3): 662-672 
Morais ICO, Brito MT, Mariz SR, Fook SML, Rabello IP, Oliveira FN Perfil epidemiológico das intoxicações medicamentosas registradas pelo Centro de Assistência e Informação Toxicológica de Campina Grande (PB) no período de 2005 a 2007. Revista Brasileira Farmácia 2008; 89 (4): 352-357

Data Sus. (2014) Disponível em:

<http://tabnet.datasus.gov.br/cgi/deftohtm.exe?sinannet/cnv/Intoxbr.def> . Acesso em: 10 de Abril, 2016 